\title{
NONOSCILLATORY DIFFERENTIAL EQUATIONS WITH RETARDED AND ADVANCED ARGUMENTS*
}

\author{
By \\ K. GOPALSAMY \\ Flinders University of South A ustralia
}

Abstract. Sufficient conditions are derived for a vector-matrix system of the form

$$
\frac{d^{n} X(t)}{d t^{n}}+(-1)^{n-1}\left[P(t) X\left(t-\tau_{1}(t)\right)+Q(t) X\left(t+\tau_{2}(t)\right)\right]=0
$$

to be nonoscillatory.

1. Introduction. We will first derive a set of sufficient conditions for scalar differential equations of the form

$$
\frac{d^{n} x(t)}{d t^{n}}+(-1)^{n-1}\left[a(t) x\left(t-\tau_{1}(t)\right)+b(t) x\left(t+\tau_{2}(t)\right)\right]=0
$$

to be nonoscillatory. Although some authors (Kusano [3], Anderson [1]) have discussed oscillatory nature of (1.1), the literature concerned with nonoscillation of equations of the form (1.1) is scarce. We will assume the following for (1.1);

$\left(\mathrm{A}_{1}\right) a, b, \tau_{1}, \tau_{2}$ are bounded continuous functions defined on $\mathbb{R}=(-\infty, \infty)$ such that for $t \in \mathbb{R}$.

$$
\begin{array}{ll}
0<a(t) \leqslant \alpha ; & 0 \leqslant t-\tau_{1}(t) \\
0 \leqslant b(t) \leqslant \beta ; & 0 \leqslant \tau_{2}(t) \\
0<\tau_{1}(t) \leqslant \sigma ; &
\end{array}
$$

where $\alpha, \beta, \sigma$ are positive constants.

$\left(\mathrm{A}_{2}\right)$ The positive constants $\alpha, \beta, \sigma$ are such that

$$
(\alpha+\beta) e^{n} \sigma^{n} / n^{n} \leqslant 1 .
$$

The following elementary observation is useful in proving our nonoscillation result. Consider a function $g:[0, \infty) \rightarrow \mathbb{R}$ defined by

$$
g(\mu)=\mu^{n}-(\alpha+\beta) e^{\mu \sigma} .
$$

* Received April 3, 1984. 
Since we have

$$
\begin{aligned}
g(0) & =-(\alpha+\beta)<0 \\
g(n / \sigma) & =(n / \sigma)^{n}-(\alpha+\beta) e^{n} \\
& \left.=(n / \sigma)^{n}\left[1-(\alpha+\beta) e^{n} \sigma^{n} / n^{n}\right] \geqslant 0 \quad \text { (by }(1.2)\right),
\end{aligned}
$$

it will follow that $g(\mu)=0$ has a positive root say $\mu^{*}$ such that

$$
\left(\mu^{*}\right)^{n}=(\alpha+\beta) e^{\sigma \mu^{*}}
$$

2. Nonoscillatory scalar systems. As it is customary we will say that (1.1) is oscillatory if and only if all solutions of (1.1) have zeros on every interval of the form $[\alpha, \infty)$ for arbitrary real constants $\alpha$ and (1.1) will be called nonoscillatory if there exists at least one solution of (1.1) having no zeros on an interval of the form $[\beta, \infty)$ for some real constant $\beta$. We can now establish the following:

THEOREM 2.1. Suppose $a, b, \tau_{1}, \tau_{2}$ in (1.1) satisfy the hypothesis $\left(\mathrm{A}_{1}\right)$ and $\left(\mathrm{A}_{2}\right)$; then (1.1) is nonoscillatory.

Proof. As one will see the proof is surprisingly simple. We consider a sequence $\left\{x_{m}(t) ; t \geqslant-\sigma ; m=0,1,2, \ldots\right\}$ defined as follows:

$$
\begin{aligned}
& x_{0}(t)=\exp \left[-\mu^{*} t\right] ; \quad t \geqslant-\sigma ; \\
& x_{m+1}(t)=\left\{\begin{array}{l}
\exp \left[-\mu^{*} t\right] ; \quad t \in[-\sigma, 0] ; \\
\int_{t}^{\infty} \frac{(s-t)}{(n-1) !}\left[a(s) x_{m}\left(s-\tau_{1}(s)\right)+b(s) x_{m}\left(s+\tau_{2}(s)\right)\right] d s,
\end{array}\right. \\
& t>0
\end{aligned}
$$

It is immediate from $(2.1)-(2.2)$ that

$$
\begin{aligned}
x_{1}(t) & \leqslant \int_{t}^{\infty} \frac{(s-t)}{(n-1) !}\left[\alpha \exp \left[-\mu^{*}(s-\sigma)\right]+\beta \exp \left[-\mu^{*}\left(s+\tau_{2}(s)\right)\right]\right] d s \\
& \leqslant(\alpha+\beta)\left(\exp \left[\mu^{*} \sigma\right]\right) \int_{t}^{\infty} \frac{(s-t)^{n \cdot 1}}{(n-1) !} \exp \left[-\mu^{*} s\right] d s \\
& \leqslant(\alpha+\beta)\left\{\exp \left[\mu^{*} \sigma\right] /\left(\mu^{*}\right)^{n}\right\} \exp \left[-\mu^{*} t\right] \\
& \leqslant e^{-\mu^{*} t} \quad\left(\text { by the choice of } \mu^{*}\right) \\
& \leqslant x_{0}(t) \quad \text { for } t>0,
\end{aligned}
$$

and hence

$$
x_{1}(t)-x_{0}(t) \leqslant 0 \text { for } t \geqslant-\sigma .
$$

From (2.2) and (2.3) one can similarly obtain

$$
x_{2}(t)-x_{1}(t) \leqslant 0 \text { for } t \geqslant-\sigma,
$$

and repeating the above procedure we derive

$$
0 \leqslant x_{m+1}(t) \leqslant x_{m}(t) \leqslant \cdots \leqslant x_{1}(t) \leqslant x_{0}(t) \text { for } t \geqslant-\sigma .
$$


The pointwise limit of the sequence $\left\{x_{m}(t)\right\}$ as $m \rightarrow \infty$ exists for $t \geqslant-\sigma$ and so we can let

$$
\lim _{m \rightarrow \infty} x_{n}(t)=x^{*}(t), \quad t \geqslant-\sigma .
$$

It will now follow by Lebesgue's dominated convergence theorem that

$$
\begin{array}{r}
x^{*}(t)=\int_{t}^{\infty} \frac{(s-t)}{(n-1) !}{ }^{n-1}\left[a(s) x^{*}\left(s-\tau_{1}(s)\right)+b(s) x^{*}\left(s+\tau_{2}(s)\right)\right] d s, \\
t>0,
\end{array}
$$

showing that $x^{*}$ is a solution of (1.1) for $t>0$. Since $x^{*}$ is the limit of a sequence of nonnegative functions, $x^{*}$ itself is nonnegative. Now it will follow from (2.7) that $x^{*}(t)>0$ on $[-\sigma, \infty)$ for if $x^{*}(t)>0$ on $[-\sigma, \tilde{t})$ and $x^{*}(\tilde{t})=0$ then (2.7) will lead to a contradiction. The result follows.

3. Nonoscillatory vector-matrix systems. Let us now consider the vector-matrix system

$$
\begin{aligned}
\frac{d^{n} X(t)}{d t^{n}}+(-1)^{n-1}\left[P(t) X\left(t-\tau_{1}(t)\right)+Q(t) X\left(t+\tau_{2}(t)\right)\right]= & , \\
& t>0,
\end{aligned}
$$

with the following assumptions:

$\left(\mathrm{A}_{3}\right) \tau_{1}, \tau_{2}$ are bounded continuous scalar functions as in $\left(\mathrm{A}_{1}\right)$.

$\left(\mathrm{A}_{4}\right) P(t), Q(t)$ are $m \times m$ matrices with nonnegative elements such that at least one element of $P(t)$ is positive and in an element wise ordering we have

$$
0 \leqslant P(t)+Q(t) \leqslant M \text { for } t \geqslant 0
$$

where $M$ is a constant $m \times m$ matrix with positive elements.

We will need the following preparation; it is well known (Perron's theorem) that $M$ will have a positive eigenvalue say $\alpha^{*}$ corresponding to which $M$ will have an eigenvector say $Z$ with positive elements. Consider now a "majorant" of (3.1) in the form

$$
\frac{d^{n} Y(t)}{d t^{n}}+(-1)^{n-1} M Y(t-\sigma)=0 ; \quad t>0 .
$$

The characteristic equation associated with (3.3) is given by

$$
\operatorname{det}\left[\lambda^{n} I+(-1)^{n-1} M e^{-\lambda \sigma}\right]=0
$$

or equivalently

$$
\operatorname{det}\left[\mu^{n} I-M e^{\mu \sigma}\right]=0, \quad \text { with } \mu=-\lambda .
$$

If $\alpha_{1}, \alpha_{2}, \ldots, \alpha_{m}$ are the eigenvalues of $M$ we have

$$
\operatorname{det}\left[\mu^{n} I-M e^{\mu \sigma}\right]=0 \Leftrightarrow \prod_{j=1}^{m}\left[\mu^{n}-\alpha_{j} e^{\mu \sigma}\right]=0 .
$$

If $\alpha_{s}=\alpha^{*}$ for some $s \in(1,2, \ldots, m)$ we can consider

$$
\mu^{n}-\alpha^{*} e^{\mu \sigma}=0
$$


in looking for real roots of (3.4). Let us now assume that

$$
\alpha^{*}(\sigma)^{n} e^{n} / n^{n} \leqslant 1 .
$$

It will then follow that (3.5) has a positive root say $\mu^{*}$ corresponding to which (3.3) will have a solution given by

$$
Y(t)=Z\left(\exp \left[-\mu^{*} t\right]\right) ; \quad t \geqslant-\sigma
$$

( $Z$ being a positive eigenvector associated with the positive eigenvalue $\mu^{*}$ of $M$ ). With this preparation we can now formulate the following for (3.1).

TheOREM 3.1. Assume that $P, Q, \tau_{1}, \tau_{2}$ satisfy the hypotheses $\left(\mathrm{A}_{3}\right)$ and $\left(\mathrm{A}_{4}\right)$. Furthermore assume that (3.6) holds. Then (3.1) is nonoscillatory.

Proof. Proof is quite similar to that of the scalar case and we provide a brief outline only. Define a sequence $\left\{X^{K}(t) ; t \geqslant-\sigma ; K=0,1,2, \ldots\right\}$ as follows:

$$
X^{K+1}(t)=\left\{\begin{array}{l}
Z\left(\exp \left[-\mu^{*} t\right]\right) ; \quad t \in[-\sigma, 0] \\
\int_{t}^{x} \frac{(s-t)}{(n-1) !}{ }^{n-1}\left[P(s) X^{(K)}\left(s-\tau_{1}(s)\right)+Q(s) X^{(K)}\left(s+\tau_{2}(s)\right)\right] d s
\end{array}\right.
$$

With a componentwise comparison, it will follow as in the scalar case (on using (3.6)),

$$
0 \leqslant X^{(K)}(t) \leqslant X^{(K-1)}(t) \leqslant \cdots \leqslant X^{(1)}(t) \leqslant X^{(0)}(t), \quad t>-\sigma,
$$

and the rest of the proof is exactly similar to that in Theorem (2.1) and we will omit further details.

We conclude with a remark that we have shown elsewhere [2] that conditions of the type in (1.2) and (3.6) are in fact necessary also for equations of the form (1.1) with (3.1) with constant coefficients to be nonoscillatory.

\section{REFERENCES}

[1] C. H. Anderson, Asymptotic oscillation results for solutions of first-order nonlinear differential-difference equations of advanced type, J. Math. Anal. Appl. 24, 430-439 (1968).

[2] K. Gopalsamy, Oscillations in linear sistems of differential-difference equations, Bull. Austral. Math. Soc. 29. $377-387$ (1984)

[3] T. Kusano, On even order functional differential equations with advanced and retarded arguments, J. Diff. Equns. 45, 75-84 (1984) 